\title{
CONTROLLABILITY OF TIME-DEPENDENT NEUTRAL STOCHASTIC FUNCTIONAL DIFFERENTIAL EQUATIONS DRIVEN BY A FRACTIONAL BROWNIAN MOTION
}

\author{
EL HASSAN LAKHEL
}

National School of Applied Sciences, Cadi Ayyad University, 46000 Safi, Morocco

\begin{abstract}
In this paper we consider the controllability of certain class of nonautonomous neutral evolution stochastic functional differential equations, with time varying delays, driven by a fractional Brownian motion in a separable real Hilbert space. Sufficient conditions for controllability are obtained by employing a fixed point approach. A practical example is provided to illustrate the viability of the abstract result of this work.
\end{abstract}

Keywords: Controllability. Neutral stochastic functional differential equations. Evolution operator. Fractional Brownian motion.

2000 Mathematics Subject Classification. 35R10, 60H15, 60G15, $60 \mathrm{~J} 65$.

\section{IntRoduction}

Controllability is one of the fundamental concepts in mathematical control theory and plays an important role in control systems. Controllability generally means that it is possible to steer a dynamical control system from an arbitrary initial state to an arbitrary final state using the set of admissible controls. If the system cannot be controlled completely then different types of controllability can be defined such as approximate, null, local null and local approximate null controllability. A standard approach is to transform the controllability problem into a fixed-point problem for an appropriate operator in a functional space. The problem of controllability for functional differential systems has been extensively studied in many papers [4, 5, 6, 11, 17, 23. For example, Sakthivel and Ren 24] studied the complete controllability of stochastic evolution equations with jumps. In [7, Balasubramaniam and Dauer discussed the controllability of semilinear stochastic delay evolution equations in Hilbert spaces.

It is known that fractional Brownian motion, with Hurst parameter $H \in(0,1)$, is a generalization of Brownian motion, it reduces to Brownian motion when $H=\frac{1}{2}$. A general theory for the infinite dimensional stochastic differential equations driven by a fractional Brownian motion ( $\mathrm{fBm}$ ) is not yet established and just a few results have been proved. In addition, in many mathematical models the claims often display long-range memories, possibly due to extreme weather, natural disasters, in some cases, many stochastic dynamical systems depend not only on present and

${ }^{1}$ Lakhel E.: e.lakhel@uca.ma 
past states, but also contain the derivatives with delays. Neutral functional differential equations are often used to describe such systems. Very recently, neutral stochastic functional differential equations driven by fractional Brownian motion have attracted the interest of many researchers. One can see [8, 12, 13] and the references therein. The literature concerning the existence and qualitative properties of solutions of time-dependent functional stochastic differential equations is very restricted and limited to a very few articles. This fact is the main motivation of our work. We mention here the recent paper by Ren et al. 21] concerning the existence of mild solutions for a class of stochastic evolution equations driven by fractional Brownian motion in Hilbert space.

Motivated by the above works, this paper is concerned with the controllability results for a class of time-dependent neutral functional stochastic differential equations described in the form:

$$
\left\{\begin{array}{l}
d[x(t)+g(t, x(t-r(t)))]=[A(t) x(t)+f(t, x(t-\rho(t)))+B u(t)] d t+\sigma(t) d B^{H}(t), t \in[0, T], \\
x(t)=\varphi(t),-\tau \leq t \leq 0 \quad \text { a.s. } \quad \tau>0,
\end{array}\right.
$$

in a real Hilbert space $X$ with inner product $<., .>$ and norm $\|$.$\| , where$ $\{A(t), t \in[0, T]\}$ is a family of linear closed operators from a space $X$ into $X$ that generates an evolution system of operators $\{U(t, s), 0 \leq s \leq t \leq T\}$. $B^{H}$ is a fractional Brownian motion on a real and separable Hilbert space $Y, r, \rho$ : $[0,+\infty) \rightarrow[0, \tau](\tau>0)$ are continuous and $f, g:[0,+\infty) \times X \rightarrow X, \sigma:$ $[0,+\infty) \rightarrow \mathcal{L}_{2}^{0}(Y, X)$, are appropriate functions. Here $\mathcal{L}_{2}^{0}(Y, X)$ denotes the space of all $Q$-Hilbert-Schmidt operators from $Y$ into $X$ (see section 2 below).

To the best of our knowledge, there is no paper which investigates the study of controllability for time-dependent neutral stochastic functional differential equations with delays driven by fractional Brownian motion. Thus, we will make the first attempt to study such problem in this paper.

Our results are inspired by the one in 10 where the existence and uniqueness of mild solutions to model (1.1) with $B=0$, is studied.

The rest of this paper is organized as follows. Section 2, recapitulate some notations, basic concepts, and basic results about fractional Brownian motion, Wiener integral over Hilbert spaces and we recall some preliminary results about evolution operator. Section 3, gives sufficient conditions to prove the controllability result for the problem (1.1). In Section 4 we give an example to illustrate the efficiency of the obtained result.

\section{Preliminaries}

2.1. Evolution families. In this subsection we introduce the notion of evolution family.

Definition 2.1. A set $\{U(t, s): 0 \leq s \leq t \leq T\}$ of bounded linear operators on a Hilbert space $X$ is called an evolution family if

(a) $U(t, s) U(s, r)=U(t, r), U(s, s)=I$ if $r \leq s \leq t$,

(b) $(t, s) \rightarrow U(t, s) x$ is strongly continuous for $t>s$.

Let $\{A(t), t \in[0, T]\}$ be a family of closed densely defined linear unbounded operators on the Hilbert space $X$ and with domain $D(A(t))$ independent of $t$, satisfying the following conditions introduced by [1]. 
There exist constants $\lambda_{0} \geq 0, \theta \in\left(\frac{\pi}{2}, \pi\right), L, K \geq 0$, and $\mu, \nu \in(0,1]$ with $\mu+\nu>1$ such that

$$
\Sigma_{\theta} \cup\{0\} \subset \rho\left(A(t)-\lambda_{0}\right), \quad\left\|R\left(\lambda, A(t)-\lambda_{0}\right)\right\| \leq \frac{K}{1+|\lambda|}
$$

and

$$
\left\|\left(A(t)-\lambda_{0}\right) R\left(\lambda, A(t)-\lambda_{0}\right)\left[R\left(\lambda_{0}, A(t)\right)-R\left(\lambda_{0}, A(s)\right)\right]\right\| \leq L|t-s|^{\mu}|\lambda|^{-\nu},
$$

for $t, s \in \mathbb{R}, \lambda \in \Sigma_{\theta}$ where $\Sigma_{\theta}:=\{\lambda \in \mathbb{C}-\{0\}:|\arg \lambda| \leq \theta\}$.

It is well known, that this assumption implies that there exists a unique evolution family $\{U(t, s): 0 \leq s \leq t \leq T\}$ on $X$ such that $(t, s) \rightarrow U(t, s) \in \mathcal{L}(X)$ is continuous for $t>s, U(\cdot, s) \in \mathcal{C}^{1}((s, \infty), \mathcal{L}(X)), \partial_{t} U(t, s)=A(t) U(t, s)$, and

$$
\left\|A(t)^{k} U(t, s)\right\| \leq C(t-s)^{-k}
$$

for $0<t-s \leq 1, k=0,1,0 \leq \alpha<\mu, x \in D\left(\left(\lambda_{0}-A(s)\right)^{\alpha}\right)$, and a constant $C$ depending only on the constants in (2.1)-(2.2). Moreover, $\partial_{s}^{+} U(t, s) x=-U(t, s) A(s) x$ for $t>s$ and $x \in D(A(s))$ with $A(s) x \in \overline{D(A(s))}$. We say that $A(\cdot)$ generates $\{U(t, s): 0 \leq s \leq t \leq T\}$. Note that $U(t, s)$ is exponentially bounded by (2.3) with $k=0$.

Remark 2.2. If $\{A(t), t \in[0, T]\}$ is a second order differential operator $A$, that is $A(t)=A$ for each $t \in[0, T]$, then $A$ generates a $C_{0}$-semigroup $\left\{e^{A t}, t \in[0, T]\right\}$.

For additional details on evolution system and their properties, we refer the reader to 25$]$.

2.2. Fractional Brownian Motion. For the convenience for the reader we recall briefly here some of the basic results of fractional Brownian motion calculus. For details of this section, we refer the reader to $[18$ and the references therein.

Let $(\Omega, \mathcal{F}, \mathbb{P})$ be a complete probability space. A standard fractional Brownian motion $(\mathrm{fBm})\left\{\beta^{H}(t), t \in \mathbb{R}\right\}$ with Hurst parameter $H \in(0,1)$ is a zero mean Gaussian process with continuous sample paths such that

$$
\mathbb{E}\left[\beta^{H}(t) \beta^{H}(s)\right]=\frac{1}{2}\left(t^{2 H}+s^{2 H}-|t-s|^{2 H}\right)
$$

for $s, t \in \mathbb{R}$. It is clear that for $H=1 / 2$, this process is a standard Brownian motion. In this paper, it is assumed that $H \in\left(\frac{1}{2}, 1\right)$.

This process was introduced by [15] and later studied by [16]. Its self-similar and long-range dependence make this process a useful driving noise in models arising in physics, telecommunication networks, finance and other fields.

Consider a time interval $[0, T]$ with arbitrary fixed horizon $T$ and let $\left\{\beta^{H}(t), t \in\right.$ $[0, T]\}$ the one-dimensional fractional Brownian motion with Hurst parameter $H \in$ $(1 / 2,1)$. It is well known that $\beta^{H}$ has the following Wiener integral representation:

$$
\beta^{H}(t)=\int_{0}^{t} K_{H}(t, s) d \beta(s),
$$

where $\beta=\{\beta(t): t \in[0, T]\}$ is a Wiener process, and $K_{H}(t ; s)$ is the kernel given by

$$
K_{H}(t, s)=c_{H} s^{\frac{1}{2}-H} \int_{s}^{t}(u-s)^{H-\frac{3}{2}} u^{H-\frac{1}{2}} d u,
$$


for $t>s$, where $c_{H}=\sqrt{\frac{H(2 H-1)}{\beta\left(2-2 H, H-\frac{1}{2}\right)}}$ and $\beta($,$) denotes the Beta function. We put$ $K_{H}(t, s)=0$ if $t \leq s$.

We will denote by $\mathcal{H}$ the reproducing kernel Hilbert space of the fBm. In fact $\mathcal{H}$ is the closure of the set of indicator functions $\left\{1_{[0 ; t]}, t \in[0, T]\right\}$ with respect to the scalar product

$$
\left\langle 1_{[0, t]}, 1_{[0, s]}\right\rangle_{\mathcal{H}}=R_{H}(t, s) .
$$

The mapping $1_{[0, t]} \rightarrow \beta^{H}(t)$ can be extended to an isometry between $\mathcal{H}$ and the first Wiener chaos and we will denote by $\beta^{H}(\varphi)$ the image of $\varphi$ by the previous isometry.

We recall that for $\psi, \varphi \in \mathcal{H}$ their scalar product in $\mathcal{H}$ is given by

$$
\langle\psi, \varphi\rangle_{\mathcal{H}}=H(2 H-1) \int_{0}^{T} \int_{0}^{T} \psi(s) \varphi(t)|t-s|^{2 H-2} d s d t .
$$

Let us consider the operator $K_{H}^{*}$ from $\mathcal{H}$ to $\mathbb{L}^{2}([0, T])$ defined by

$$
\left(K_{H}^{*} \varphi\right)(s)=\int_{s}^{T} \varphi(r) \frac{\partial K}{\partial r}(r, s) d r
$$

We refer to [18] for the proof of the fact that $K_{H}^{*}$ is an isometry between $\mathcal{H}$ and $L^{2}([0, T])$. Moreover for any $\varphi \in \mathcal{H}$, we have

$$
\beta^{H}(\varphi)=\int_{0}^{T}\left(K_{H}^{*} \varphi\right)(t) d \beta(t) .
$$

It follows from [18] that the elements of $\mathcal{H}$ may be not functions but distributions of negative order. In the case $H>\frac{1}{2}$, the second partial derivative of the covariance function

$$
\frac{\partial R_{H}}{\partial t \partial s}=\alpha_{H}|t-s|^{2 H-2}
$$

where $\alpha_{H}=H(2 H-2)$, is integrable, and we can write

$$
R_{H}(t, s)=\alpha_{H} \int_{0}^{t} \int_{0}^{s}|u-v|^{2 H-2} d u d v .
$$

In order to obtain a space of functions contained in $\mathcal{H}$, we consider the linear space $|\mathcal{H}|$ generated by the measurable functions $\psi$ such that

$$
\|\psi\|_{|\mathcal{H}|}^{2}:=\alpha_{H} \int_{0}^{T} \int_{0}^{T}|\psi(s)\|\psi(t)\| s-t|^{2 H-2} d s d t<\infty,
$$

where $\alpha_{H}=H(2 H-1)$. The space $|\mathcal{H}|$ is a Banach space with the norm $\|\psi\|_{|\mathcal{H}|}$ and we have the following inclusions (see [18]).

\section{Lemma 2.3.}

$$
\mathbb{L}^{2}([0, T]) \subseteq \mathbb{L}^{1 / H}([0, T]) \subseteq|\mathcal{H}| \subseteq \mathcal{H}
$$

and for any $\varphi \in \mathbb{L}^{2}([0, T])$, we have

$$
\|\psi\|_{|\mathcal{H}|}^{2} \leq 2 H T^{2 H-1} \int_{0}^{T}|\psi(s)|^{2} d s .
$$

Let $X$ and $Y$ be two real, separable Hilbert spaces and let $\mathcal{L}(Y, X)$ be the space of bounded linear operator from $Y$ to $X$. For the sake of convenience, we shall use the same notation to denote the norms in $X, Y$ and $\mathcal{L}(Y, X)$. Let $Q \in \mathcal{L}(Y, Y)$ be an operator defined by $Q e_{n}=\lambda_{n} e_{n}$ with finite $\operatorname{trace} \operatorname{tr} Q=\sum_{n=1}^{\infty} \lambda_{n}<\infty$. 
where $\lambda_{n} \geq 0(n=1,2 \ldots)$ are non-negative real numbers and $\left\{e_{n}\right\} \quad(n=1,2 \ldots)$ is a complete orthonormal basis in $Y$. Let $B^{H}=\left(B^{H}(t)\right)$ be $Y$ - valued fbm on $(\Omega, \mathcal{F}, \mathbb{P})$ with covariance $Q$ as

$$
B^{H}(t)=B_{Q}^{H}(t)=\sum_{n=1}^{\infty} \sqrt{\lambda_{n}} e_{n} \beta_{n}^{H}(t)
$$

where $\beta_{n}^{H}$ are real, independent fBm's. This process is Gaussian, it starts from 0 , has zero mean and covariance:

$$
E\left\langle B^{H}(t), x\right\rangle\left\langle B^{H}(s), y\right\rangle=R(s, t)\langle Q(x), y\rangle \text { for all } x, y \in Y \text { and } t, s \in[0, T] .
$$

In order to define Wiener integrals with respect to the $Q$ - $\mathrm{fBm}$, we introduce the space $\mathcal{L}_{2}^{0}:=\mathcal{L}_{2}^{0}(Y, X)$ of all $Q$-Hilbert-Schmidt operators $\psi: Y \rightarrow X$. We recall that $\psi \in \mathcal{L}(Y, X)$ is called a $Q$-Hilbert-Schmidt operator, if

$$
\|\psi\|_{\mathcal{L}_{2}^{0}}^{2}:=\sum_{n=1}^{\infty}\left\|\sqrt{\lambda_{n}} \psi e_{n}\right\|^{2}<\infty,
$$

and that the space $\mathcal{L}_{2}^{0}$ equipped with the inner product $\langle\varphi, \psi\rangle_{\mathcal{L}_{2}^{0}}=\sum_{n=1}^{\infty}\left\langle\varphi e_{n}, \psi e_{n}\right\rangle$ is a separable Hilbert space.

Now, let $\phi(s) ; s \in[0, T]$ be a function with values in $\mathcal{L}_{2}^{0}(Y, X)$, such that $\sum_{n=1}^{\infty}\left\|K^{*} \phi Q^{\frac{1}{2}} e_{n}\right\|_{\mathcal{L}_{2}^{0}}^{2}<\infty$. The Wiener integral of $\phi$ with respect to $B^{H}$ is defined by

$$
\int_{0}^{t} \phi(s) d B^{H}(s)=\sum_{n=1}^{\infty} \int_{0}^{t} \sqrt{\lambda_{n}} \phi(s) e_{n} d \beta_{n}^{H}(s)=\sum_{n=1}^{\infty} \int_{0}^{t} \sqrt{\lambda_{n}}\left(K_{H}^{*}\left(\phi e_{n}\right)(s) d \beta_{n}(s)\right.
$$

where $\beta_{n}$ is the standard Brownian motion used to present $\beta_{n}^{H}$ as in (2.5).

Now, we end this subsection by stating the following result which is fundamental to prove our result.

Lemma 2.4. 10] Suppose that $\sigma:[0, T] \rightarrow \mathcal{L}_{2}^{0}(Y, X)$ satisfies $\sup _{t \in[0, T]}\|\sigma(t)\|_{\mathcal{L}_{2}^{0}}^{2}<\infty$, and Suppose that $\{U(t, s), 0 \leq s \leq t \leq T\}$ is an evolution system of operators satisfying $\|U(t, s)\| \leq M e^{-\beta(t-s)}$, for some constants $\beta>0$ and $M \geq 1$ for all $t \geq s$. Then, we have

$$
\mathbb{E}\left\|\int_{0}^{t} U(t, s) \sigma(s) d B^{H}(s)\right\|^{2} \leq C M^{2} t^{2 H}\left(\sup _{t \in[0, T]}\|\sigma(t)\|_{\mathcal{L}_{2}^{0}}\right)^{2} .
$$

Remark 2.5. Thanks to Lemma 2.4, the stochastic integral

$$
Z(t)=\int_{0}^{t} U(t, s) \sigma(s) d B^{H}(s), \quad t \in[0, T]
$$

is well-defined.

\section{Controllability Result}

Henceforth we will assume that the family $\{A(t), t \in[0, T]\}$ of linear operators generates an evolution system of operators $\{U(t, s), 0 \leq s \leq t \leq T\}$. In this section we derive controllability conditions for time-dependent neutral stochastic functional differential equations with variable delays driven by a fractional Brownian motion in a real separable Hilbert space. Before starting, we introduce the concept of a mild 
solution of the problem (1.1) and controllability of neutral stochastic functional differential equation.

Definition 3.1. An $X$-valued process $\{x(t), t \in[-\tau, T]\}$, is called a mild solution of equation (1.1) if

i) $x(.) \in \mathcal{C}\left([-\tau, T], \mathbb{L}^{2}(\Omega, X)\right)$,

ii) $x(t)=\varphi(t),-\tau \leq t \leq 0$.

iii) For arbitrary $t \in[0, T]$, we have

$$
\begin{aligned}
x(t) & =U(t, 0)(\varphi(0)+g(0, \varphi(-r(0))))-g(t, x(t-r(t))) \\
& -\int_{0}^{t} A U(t, s) g(s, x(s-r(s))) d s+\int_{0}^{t} U(t, s) f(s, x(s-\rho(s))) d s \\
& +\int_{0}^{t} U(t, s)(B u)(s) d s+\int_{0}^{t} U(t, s) \sigma(s) d B^{H}(s), \quad \mathbb{P}-a . s .
\end{aligned}
$$

Definition 3.2. The system (1.1) is said to be controllable on the interval $[-\tau, T]$, if for every initial stochastic process $\varphi$ defined $[-\tau, 0]$, there exists a stochastic control $u \in L^{2}([0, T], U)$ such that the mild solution $x($.$) of (1.1) satisfies x(T)=x_{1}$, where $x_{1}$ and $T$ are the preassigned terminal state and time, respectively.

We will study the problem (1.1) under the following assumptions:

$(\mathcal{H} .1)$ i) The evolution family is exponentially stable, that is, there exist two constants $\beta>0$ and $M \geq 1$ such that

$$
\|U(t, s)\| \leq M e^{-\beta(t-s)}, \quad \text { for all } t \geq s,
$$

ii) There exist a constant $M_{*}>0$ such that

$$
\left\|A^{-1}(t)\right\| \leq M_{*} \quad \text { for all } t \in[0, T] .
$$

$(\mathcal{H} .2)$ The maps $f, g:[0, T] \times X \rightarrow X$ are continuous functions and there exist two positive constants $C_{1}$ and $C_{2}$, such that for all $t \in[0, T]$ and $x, y \in X$ :

i) $\|f(t, x)-f(t, y)\| \vee\|g(t, x)-g(t, y)\| \leq C_{1}\|x-y\|$.

ii) $\|f(t, x)\|^{2} \vee\left\|A^{k}(t) g(t, x)\right\|^{2} \leq C_{2}\left(1+\|x\|^{2}\right), \quad k=0,1$.

$\left(\begin{array}{ll}\mathcal{H} .3 & i\end{array}\right)$ There exists a positive constant $L_{*}$ such that $L^{*} M_{*}<\frac{1}{\sqrt{6}}$, and

$$
\|A(t) g(t, x)-A(t) g(t, y)\| \leq L_{*}\|x-y\|,
$$

for all $t \in[0, T]$ and $x, y \in X$.

ii) The function $g$ is continuous in the quadratic mean sense: for all $x(.) \in$ $\mathcal{C}\left([0, T], L^{2}(\Omega, X)\right)$, we have

$$
\lim _{t \longrightarrow s} \mathbb{E}\|g(t, x(t))-g(s, x(s))\|^{2}=0 .
$$

$(\mathcal{H} .4) \quad i)$ The map $\sigma:[0, T] \longrightarrow \mathcal{L}_{2}^{0}(Y, X)$ is bounded, that is : there exists a positive constant $L$ such that $\|\sigma(t)\|_{\mathcal{L}_{2}^{0}(Y, X)} \leq L$ uniformly in $t \in[0, T]$.

ii) Moreover, we assume that the initial data $\varphi=\{\varphi(t):-\tau \leq t \leq 0\}$ satisfies $\varphi \in \mathcal{C}\left([-\tau, 0], \mathbb{L}^{2}(\Omega, X)\right)$.

$(\mathcal{H} .5)$ The linear operator $W$ from $U$ into $X$ defined by

$$
W u=\int_{0}^{T} U(T, s) B u(s) d s
$$


has an inverse operator $W^{-1}$ that takes values in $L^{2}([0, T], U) \backslash \operatorname{ker} W$, where $\operatorname{ker} W=\left\{x \in L^{2}([0, T], U), W x=0\right\}$ (see [14]), and there exists finite positive constants $M_{b}, M_{w}$ such that $\|B\| \leq M_{b}$ and $\left\|W^{-1}\right\| \leq M_{w}$.

The main result of this paper is given in the next theorem.

Theorem 3.3. Suppose that $(\mathcal{H} .1)-(\mathcal{H} .5)$ hold. Then, the system (1.1) is controllable on $[-\tau, T]$.

Proof. Fix $T>0$ and let $\mathcal{B}_{T}:=\mathcal{C}\left([-\tau, T], \mathbb{L}^{2}(\Omega, X)\right.$ be the Banach space of all continuous functions from $[-\tau, T]$ into $\mathbb{L}^{2}(\Omega, X)$, equipped with the supremum norm $\|\xi\|_{\mathcal{B}_{T}}=\sup _{u \in[-\tau, T]}\left(\mathbb{E}\|\xi(u)\|^{2}\right)^{1 / 2}$ and let us consider the set

$$
S_{T}=\left\{x \in \mathcal{B}_{T}: x(s)=\varphi(s), \text { for } s \in[-\tau, 0]\right\}
$$

$S_{T}$ is a closed subset of $\mathcal{B}_{T}$ provided with the norm $\|\cdot\|_{\mathcal{B}_{T}}$.

Using the hypothesis $(\mathcal{H} .5)$ for an arbitrary function $x($.$) , define the stochastic$ control

$$
\begin{aligned}
u(t) & =W^{-1}\left\{x_{1}-U(T, 0)(\varphi(0)+g(0, \varphi(-r(0))))+g(T, x(T-r(T)))\right. \\
& +\quad \int_{0}^{T} A U(T, s) g(s, x(s-r(s))) d s-\int_{0}^{T} U(T, s) f(s, x(s-\rho(s)) d s \\
& -\int_{0}^{T} U(T, s) \sigma(s) d B^{H}(s) .
\end{aligned}
$$

We will now show that using this control that the operator $\psi$ on $S_{T}(\varphi)$ defined by $\psi(x)(t)=\varphi(t)$ for $t \in[-\tau, 0]$ and for $t \in[0, T]$

$$
\begin{aligned}
\psi(x)(t) & =U(t, 0)(\varphi(0)+g(0, \varphi(-r(0))))-g(t, x(t-r(t)))-\int_{0}^{t} U(t, s) A(s) g(s, x(s-r(s))) d s \\
& +\int_{0}^{t} U(t, s) f(s, x(s-\rho(s))) d s+\int_{0}^{t} U(t, \nu) \sigma(s) d B^{H}(s) \\
& +\int_{0}^{t} U(t, \nu) B W^{-1}\left\{x_{1}-U(T, 0)(\varphi(0)+g(0, \varphi(-r(0))))+g(T, x(T-r(T)))\right. \\
& +\int_{0}^{T} U(T, s) A(s) g(s, x(s-r(s))) d s-\int_{0}^{T} U(T, s) f(s, x(s-\rho(s)) d s \\
& \left.-\int_{0}^{T} U(T, s) \sigma(s) d B^{H}(s)\right\} d \nu
\end{aligned}
$$

has a fixed point. This fixed point is then a solution of (1.1). Clearly, $\psi(x)(T)=x_{1}$, which implies that the system (1.1) is controllable.

For better readability, we break the proof into sequence of steps.

Step 1: $\psi$ is well defined. Let $x \in S_{T}(\varphi)$ and $t \in[0, T]$, we are going to show that each function $\psi(x)($.$) is continuous on [0, T]$ in the $\mathbb{L}^{2}(\Omega, X)$-sense. 
Let $0<t<T$ and $|h|$ be sufficiently small. Then for any fixed $x \in S_{T}$, we have

$$
\begin{aligned}
\mathbb{E} \| \psi(x)(t+ & h)-\psi(x)(t)\left\|^{2} \leq 6 \mathbb{E}\right\|(U(t+h, 0)-U(t, 0))(\varphi(0)+g(0, \varphi(-r(0)))) \|^{2} \\
& +6 \mathbb{E}\|g(t+h, x(t+h-r(t+h)))-g(t, x(t-r(t)))\|^{2} \\
& +6 \mathbb{E} \| \int_{0}^{t+h} U(t+h, s) A(s) g\left(s, x(s-r(s)) d s-\int_{0}^{t} U(t, s) A(s) g\left(s, x(s-r(s)) d s \|^{2}\right.\right. \\
& +6 \mathbb{E}\left\|\int_{0}^{t+h} U(t+h, s) f(s, x(s-\rho(s))) d s-\int_{0}^{t} U(t, s) f(s, x(s-\rho(s))) d s\right\|^{2} \\
& +6 \mathbb{E}\left\|\int_{0}^{t+h} U(t+h, s) \sigma(s) d B^{H}(s)-\int_{0}^{t} U(t, s) \sigma(s) d B^{H}(s)\right\|^{2} \\
& +6 \mathbb{E} \| \int_{0}^{t+h} U(t+h, \nu) B W^{-1}\left\{x_{1}-U(T, 0)(\varphi(0)+g(0, \varphi(-r(0))))\right. \\
& +g(T, x(T-r(T)))+\int_{0}^{T} U(T, s) A(s) g(s, x(s-r(s))) d s \\
& -\int_{0}^{T} U(T, s) f\left(s, x(s-\rho(s)) d s-\int_{0}^{T} U(T, s) \sigma(s) d B^{H}(s)\right\} d \nu \\
& -\int_{0}^{t} U(t, \nu) B W^{-1}\left\{x_{1}-U(T, 0)(\varphi(0)+g(0, \varphi(-r(0))))\right. \\
& \left.+g(T, x(T-r(T)))-\int_{0}^{T} U(T, s) \sigma(s) d B^{H}(s)\right\} d \nu \\
& =6 \sum_{1 \leq i \leq 6} \mathbb{E}\left\|I_{i}(t+h)-I_{i}(t)\right\|^{2} .
\end{aligned}
$$

From Definition 2.1, we obtain

$$
\lim _{h \longrightarrow 0}(U(t+h, 0)-U(t, 0))(\varphi(0)+g(0, \varphi(-r(0))))=0 .
$$

From $(\mathcal{H} .1)$, we have

$\|(U(t+h, 0)-U(t, 0))(\varphi(0)+g(0, \varphi(-r(0))))\| \leq M e^{-\beta t}\left(e^{-\beta h}+1\right)\|\varphi(0)+g(0, \varphi(-r(0)))\| \in L^{2}(\Omega)$.

Then we conclude by the Lebesgue dominated theorem that

$$
\lim _{h \longrightarrow 0} \mathbb{E}\left\|I_{1}(t+h)-I_{1}(t)\right\|^{2}=0 .
$$

Moreover, assumption ( $\mathcal{H} .2)$ ensures that

$$
\lim _{h \longrightarrow 0} \mathbb{E}\left\|I_{2}(t+h)-I_{2}(t)\right\|^{2}=0 .
$$

To show that the third term $I_{3}(h)$ is continuous, we suppose $h>0$ (similar calculus for $h<0)$. We have

$$
\begin{aligned}
\left\|I_{3}(t+h)-I_{3}(t)\right\| \leq & \left\|\int_{0}^{t}(U(t+h, s)-U(t, s)) A(s) g(s, x(s-r(s))) d s\right\| \\
& +\| \int_{t}^{t+h}(U(t, s) g(s, x(s-r(s))) d s \| \\
\leq & I_{31}(h)+I_{32}(h) .
\end{aligned}
$$

By Hölder's inequality, we have

$$
\left.\mathbb{E}\left\|I_{31}(h)\right\| \leq t \mathbb{E} \int_{0}^{t} \| U(t+h, s)-U(t+h, s)\right) A(s) g\left(s, x(s-r(s)) \|^{2} d s .\right.
$$


By Definition 2.1, we obtain

$$
\lim _{h \longrightarrow 0}(U(t+h, s)-U(t, s)) A(s) g(s, x(s-r(s)))=0 .
$$

From $(\mathcal{H} .1)$ and $(\mathcal{H} .2)$, we have

$\| U(t+h, s)-U(t, s)) A(s) g\left(s, x(s-r(s))\left\|\leq C_{2} M e^{-\beta(t-s)}\left(e^{-\beta h}+1\right)\right\| A(s) g\left(s, x(s-r(s)) \| \in L^{2}(\Omega)\right.\right.$.

Then we conclude by the Lebesgue dominated theorem that

$$
\lim _{h \rightarrow 0} \mathbb{E}\left\|I_{31}(h)\right\|^{2}=0 \text {. }
$$

So, estimating as before. By using $(\mathcal{H} .1)$ and $(\mathcal{H} .2)$, we get

$$
\mathbb{E}\left\|I_{32}(h)\right\|^{2} \leq \frac{M^{2} C_{2}\left(1-e^{-2 \beta h}\right)}{2 \beta} \int_{t}^{t+h}\left(1+\mathbb{E}\|x(s-r(s))\|^{2}\right) d s .
$$

Thus,

$$
\lim _{h \rightarrow 0} \mathbb{E}\left\|I_{32}(h)\right\|^{2}=0 .
$$

For the fourth term $I_{4}(h)$, we suppose $h>0$ (similar calculus for $h<0$ ). We have

$$
\begin{aligned}
\left\|I_{4}(t+h)-I_{4}(t)\right\| \leq & \left\|\int_{0}^{t}(U(t+h, s)-U(t, s)) f(s, x(s-\rho(s))) d s\right\| \\
& +\| \int_{t}^{t+h}(U(t, s) f(s, x(s-\rho(s))) d s \| \\
\leq & I_{41}(h)+I_{42}(h) .
\end{aligned}
$$

By Hölder's inequality, we have

$$
\left.\mathbb{E}\left\|I_{41}(h)\right\| \leq t \mathbb{E} \int_{0}^{t} \| U(t+h, s)-U(t, s)\right) f\left(s, x(s-\rho(s)) \|^{2} d s .\right.
$$

Again exploiting properties of Definition 2.1, we obtain

$$
\lim _{h \longrightarrow 0}(U(t+h, s)-U(t, s)) f(s, x(s-\rho(s)))=0,
$$

and

$\| U(t+h, s)-U(t, s)) f\left(s, x(s-\rho(s))\left\|\leq M e^{-\beta(t-s)}\left(e^{-\beta h}+1\right)\right\| f\left(s, x(s-\rho(s)) \| \in L^{2}(\Omega)\right.\right.$.

Then we conclude by the Lebesgue dominated theorem that

$$
\lim _{h \rightarrow 0} \mathbb{E}\left\|I_{41}(h)\right\|^{2}=0 .
$$

On the other hand, by $(\mathcal{H} .1),(\mathcal{H} .2)$, and the Hölder's inequality, we have

$$
\mathbb{E}\left\|I_{42}(h)\right\| \leq \frac{M^{2} C_{2}\left(1-e^{-2 \beta h}\right)}{2 \beta} \int_{t}^{t+h}\left(1+\mathbb{E}\|x(s-\rho(s))\|^{2}\right) d s .
$$

Thus

$$
\lim _{h \rightarrow 0} I_{42}(h)=0 .
$$

Now, for the term $I_{5}(h)$, we have

$$
\begin{aligned}
\left\|I_{5}(t+h)-I_{5}(t)\right\| & \leq \| \int_{0}^{t}\left(U(t+h, s)-U(t, s) \sigma(s) d B^{H}(s) \|\right. \\
& +\left\|\int_{t}^{t+h} U(t+h, s) \sigma(s) d B^{H}(s)\right\|
\end{aligned}
$$




$$
\leq I_{51}(h)+I_{52}(h) .
$$

By Lemma 2.4 we get that

$$
E\left\|I_{51}(h)\right\|^{2} \leq 2 H t^{2 H-1} \int_{0}^{t}\|[U(t+h, s)-U(t, s)] \sigma(s)\|_{\mathcal{L}_{2}^{0}}^{2} d s .
$$

Since

$$
\lim _{h \rightarrow 0}\|[U(t+h, s)-U(t, s)] \sigma(s)\|_{\mathcal{L}_{2}^{0}}^{2}=0
$$

and

$$
\|\left(U(t+h, s)-U(t, s) \sigma(s) \|_{\mathcal{L}_{2}^{0}} \leq M L e^{-\beta(t-s)} e^{-\beta h+1} \in \mathbb{L}^{1}([0, T], d s),\right.
$$

we conclude, by the dominated convergence theorem that,

$$
\lim _{h \rightarrow 0} \mathbb{E}\left\|I_{51}(h)\right\|^{2}=0 .
$$

Again by Lemma 2.4, we get that

$$
\mathbb{E}\left\|I_{52}(h)\right\|^{2} \leq \frac{2 H t^{2 H-1} L M^{2}\left(1-e^{-2 \beta h}\right)}{2 \beta} .
$$

Thus,

$$
\lim _{h \rightarrow 0} \mathbb{E}\left|I_{52}(h)\right|^{2}=0 .
$$

For the estimation of term $I_{6}$, we have

$$
\begin{aligned}
\mathbb{E}\left\|I_{6}(h)\right\|^{2} \leq & 2 \mathbb{E} \| \int_{t}^{t+h} U(t+h, \nu) B W^{-1}\left\{x_{1}-U(T, 0)(\varphi(0)+g(0, \varphi(-r(0))))\right. \\
& +g(T, x(T-r(T)))+\int_{0}^{T} U(T, s) A(s) g(s, x(s-r(s))) d s \\
& -\int_{0}^{T} U(T, s) f\left(s, x(s-\rho(s)) d s-\int_{0}^{T} U(T, s) \sigma(s) d B^{H}(s) \|\right. \\
& +2 \mathbb{E} \| \int_{0}^{t}(U(t+h, \nu)-U(t, \nu)) B W^{-1}\left\{x_{1}-U(T, 0)(\varphi(0)+g(0, \varphi(-r(0))))\right. \\
& +g(T, x(T-r(T)))+\int_{0}^{T} U(T, s) A(s) g(s, x(s-r(s))) d s \\
& -\int_{0}^{T} U(T, s) f\left(s, x(s-\rho(s)) d s-\int_{0}^{T} U(T, s) \sigma(s) d B^{H}(s)\right\} d \nu \| \\
& \leq 2\left[\mathbb{E}\left\|I_{6,1}(h)\right\|^{2}+\mathbb{E}\left\|I_{6,2}(h)\right\|^{2}\right] .
\end{aligned}
$$

Let's first deal with $I_{6,1}(h)$, it follows from the conditions $(\mathcal{H} .1)-(\mathcal{H} .5)$ that

$$
\begin{aligned}
\mathbb{E}\left\|I_{6,1}(h)\right\|^{2} & \leq 6 M^{2} M_{b}^{2} M_{w}^{2} \int_{t}^{t+h}\left\{\mathbb{E}\left\|x_{1}\right\|^{2}+M^{2} \mathbb{E}\|\varphi(0)+g(0, \varphi(-r(0)))\|^{2}\right. \\
& +M_{*}^{2} C_{2} T\left(1+\sup _{s \in[-\tau, T]} \mathbb{E}\|x(s)\|^{2}\right)+M^{2} T C_{2}\left(1+\sup _{s \in[-\tau, T]} \mathbb{E}\|x(s)\|^{2}\right) \\
& \left.+M^{2} T C_{2}\left(1+\sup _{s \in[-\tau, T]} \mathbb{E}\|x(s)\|^{2}\right)+2 M^{2} H T^{2 H-1} \int_{0}^{T}\|\sigma(s)\|_{\mathcal{L}_{2}^{0}}^{2} d s\right\} d \nu .
\end{aligned}
$$

It results that

$$
\lim _{h \rightarrow 0} \mathbb{E}\left\|I_{6,1}(h)\right\|^{2}=0 .
$$


In a similar way, we have

$$
\begin{aligned}
\mathbb{E}\left\|I_{6,2}(h)\right\|^{2} & \leq 6 M_{b}^{2} M_{w}^{2} \int_{0}^{t}\|U(t+h, \nu)-U(t, \nu)\|^{2}\left\{\mathbb{E}\left\|x_{1}\right\|^{2}\right. \\
& +M^{2} \mathbb{E}\|\varphi(0)+g(0, \varphi(-r(0)))\|^{2}+M_{*}^{2} C_{2}\left(1+\mathbb{E}\|x\|^{2}\right) \\
& +M^{2} T^{2} C_{2}\left(1+\mathbb{E}\|x\|^{2}\right)+M^{2} T^{2} C_{2}\left(1+\mathbb{E}\|x\|^{2}\right) \\
& \left.+2 M^{2} H T^{2 H-1} \int_{0}^{T}\|\sigma(s)\|_{\mathcal{L}_{2}^{0}}^{2} d s\right\} d \nu .
\end{aligned}
$$

Since

$$
\begin{aligned}
& \|U(t+h, \nu)-U(t, \nu)\|^{2}\left\{\mathbb{E}\left\|x_{1}\right\|^{2}+M^{2} \mathbb{E}\|\varphi(0)+g(0, \varphi(-r(0)))\|^{2}+M_{*}^{2} C_{4}\left(1+\sup _{s \in[-\tau, T]} \mathbb{E}\|x(s)\|^{2}\right)\right. \\
& +M^{2} T^{2} C_{2}\left(1+\sup _{s \in[-\tau, T]} \mathbb{E}\|x(s)\|^{2}\right)+M^{2} T^{2} C_{2}\left(1+\sup _{s \in[-\tau, T]} \mathbb{E}\|x(s)\|^{2}\right) \\
& \left.+2 M^{2} H T^{2 H-1} \int_{0}^{T}\|\sigma(s)\|_{\mathcal{L}_{2}^{0}}^{2} d s\right\} \\
& \leq 4 M^{2}\left\{\mathbb{E}\left\|x_{1}\right\|^{2}+M^{2} \mathbb{E}\|\varphi(0)+g(0, \varphi(-r(0)))\|^{2}\right. \\
& +M_{*}^{2} C_{2}\left(1+\sup _{s \in[-\tau, T]} \mathbb{E}\|x(s)\|^{2}\right)+2 M^{2} T^{2} C_{2}\left(1+\sup _{s \in[-\tau, T]} \mathbb{E}\|x(s)\|^{2}\right) \\
& \left.\left.+2 M^{2} H T^{2 H-1} \int_{0}^{T}\|\sigma(s)\|_{\mathcal{L}_{2}^{0}}^{2} d s\right\} \in L^{1}([0, T], d s]\right),
\end{aligned}
$$

we conclude, by the dominated convergence theorem that,

$$
\lim _{h \rightarrow 0} \mathbb{E}\left\|I_{6,2}(h)\right\|^{2}=0 .
$$

The above arguments show that $\lim _{h \rightarrow 0} \mathbb{E}\|\psi(x)(t+h)-\psi(x)(t)\|^{2}=0$. Hence, we conclude that the function $t \rightarrow \psi(x)(t)$ is continuous on $[0, T]$ in the $\mathbb{L}^{2}$-sense.

Step 2: Now, we are going to show that $\psi$ is a contraction mapping in $S_{T_{1}}(\varphi)$ with some $T_{1} \leq T$ to be specified later. Let $x, y \in S_{T}(\varphi)$, then for any fixed $t \in[0, T]$, we have

$$
\begin{aligned}
\mathbb{E} \quad & \|\psi(x)(t)-\psi(y)(t)\|^{2} \\
\leq & 6\left\|A(t)^{-1}\right\|^{2} \mathbb{E}\|A(t) g(t, x(t-r(t)))-A(t) g(t, y(t-r(t)))\|^{2} \\
& +6 \mathbb{E}\left\|\int_{0}^{t} U(t, s) A(s)(g(s, x(s-r(s)))-g(s, y(s-r(s)))) d s\right\|^{2} \\
& +6 \mathbb{E}\left\|\int_{0}^{t} U(t, s)(f(s, x(s-\rho(s)))-f(s, y(s-\rho(s)))) d s\right\|^{2} \\
& +6 \mathbb{E}\left\|\int_{0}^{t} U(t, \nu) B W^{-1}[g(T, x(T-r(T)))-g(T, y(T-r(T)))] d \nu\right\|^{2} \\
& \left.+6 \mathbb{E} \| \int_{0}^{t} U(t, \nu) B W^{-1} \int_{0}^{T} U(T, s) A(s)[g(s, x(s-r(s)))-g(s, y(s-r(s)))] d s\right] d \nu \|^{2} \\
& +6 \mathbb{E}\left\|\int_{0}^{t} U(t, \nu) B W^{-1} \int_{0}^{T} U(T, s)[f(s, x(s-\rho(s)))-f(s, y(s-\rho(s)))] d s d \nu\right\|^{2} .
\end{aligned}
$$


E. LAKHEL

By assumptions combined with Hölder's inequality, we get that

$$
\begin{aligned}
\mathbb{E}\|\psi(x)(t)-\psi(y)(t)\|^{2} & \leq 6 L_{*}^{2} M_{*}^{2} \sup _{s \in[-\tau, t]} \mathbb{E}\|x(t-r)-y(t-r)\|^{2} \\
& +6 M^{2} L_{*}^{2} \frac{1-e^{-2 \beta t}}{2 \beta} t \sup _{s \in[-\tau, t]} \mathbb{E}\|x(s)-y(s)\|^{2} \\
& +6 M^{2} C_{1}^{2} \frac{1-e^{-2 \beta t}}{2 \beta} t \sup _{s \in[-\tau, t]} \mathbb{E}\|x(s)-y(s)\|^{2} \\
& +6 \mathbf{t} M^{2} M_{b}^{2} M_{w}^{2}\left[C_{1}^{2} \mathbb{E}\|x(T-r(T))-y(T-r(T))\|^{2}\right. \\
& +L_{*}^{2} M^{2} T^{2} \sup _{s \in[-\tau, t]} \mathbb{E}\|x(s-r(s))-y(s-r(s))\|^{2} \\
& +T^{2} M^{2} C_{1}^{2} \sup _{s \in[-\tau, t]} \mathbb{E}\|x(s)-y(s)\|^{2} .
\end{aligned}
$$

Hence

$$
\sup _{s \in[-\tau, T]} \mathbb{E}\|\psi(x)(s)-\psi(y)(s)\|^{2} \leq \gamma(t) \sup _{s \in[-\tau, T]} \mathbb{E}\|x(s)-y(s)\|^{2},
$$

where

$$
\begin{aligned}
\gamma(t)= & 6\left[\| L_{*}^{2} M_{*}^{2}+M^{2} L_{*}^{2} \frac{1-e^{-2 \beta t}}{2 \beta} t+M^{2} C_{1}^{2} \frac{1-e^{-2 \beta t}}{2 \beta} t\right. \\
& +\mathbf{t} M^{2} M_{b}^{2} M_{w}^{2}\left(C_{1}^{2}+L_{*}^{2} M^{2} T^{2}+T^{2} M^{2} C_{1}^{2}\right] .
\end{aligned}
$$

By condition ( $\mathcal{H} .3$ ), we have $\gamma(0)=6 L_{*}^{2} M_{*}^{2}<1$. Then there exists $0<T_{1} \leq T$ such that $0<\gamma\left(T_{1}\right)<1$ and $\psi$ is a contraction mapping on $S_{T_{1}}$ and therefore has a unique fixed point, which is a mild solution of equation (1.1) on $\left[-\tau, T_{1}\right]$. This procedure can be repeated in order to extend the solution to the entire interval $[-\tau, T]$ in finitely many steps. Clearly, $(\psi x)(T)=x_{1}$ which implies that the system (1.1) is controllable on $[-\tau, T]$. This completes the proof.

\section{An illustrative Example}

In recent years, the interest in neutral systems has been growing rapidly due to their successful applications in practical fields such as physics, chemical technology, bioengineering, and electrical networks. We consider the following stochastic partial neutral functional differential equation with finite delays $\tau_{1}$ and $\tau_{2}\left(0 \leq \tau_{i} \leq \tau<\right.$ $\infty, i=1,2)$ :

$$
\left\{\begin{aligned}
d\left[u(t, \zeta)+g_{1}\left(t, u\left(t-\tau_{1}, \zeta\right)\right)\right] & =\left[\frac{\partial^{2}}{\partial^{2} \zeta} u(t, \zeta)+b(t, \zeta) u(t, \zeta)+f_{1}\left(t, u\left(t-\tau_{2}, \zeta\right)\right)\right. \\
& +v(t, \xi)] d t+\sigma(t) d B^{H}(t), 0 \leq t \leq T, 0 \leq \zeta \leq \pi, \\
u(t, 0)=u(t, \pi)=0, \quad 0 \leq t \leq T, & \\
u(t, \zeta)=\varphi(t, \zeta), \quad t \in[-\tau, 0], & 0 \leq \zeta \leq \pi,
\end{aligned}\right.
$$

where $B^{H}$ is a fractional Brownian motion, $b(t, \zeta)$ is a continuous function and is uniformly Hölder continuous in $t, f_{1}, g_{1}: \mathbb{R}^{+} \times \mathbb{R} \longrightarrow \mathbb{R}$ are continuous functions. 
To study this system, we consider the space $X=L^{2}([0, \pi])$ and the operator $A$ : $D(A) \subset X \longrightarrow X$ given by $A y=y^{\prime \prime}$ with

$$
\left.D(A)=\left\{y \in X: y^{\prime \prime} \in X, \quad y(0)\right)=y(\pi)=0\right\} .
$$

It is well known that $A$ is the infinitesimal generator of an analytic semigroup $\{T(t)\}_{t \geq 0}$ on $X$. Furthermore, $A$ has discrete spectrum with eigenvalues $-n^{2}, n \in \mathbb{N}$ and the corresponding normalized eigenfunctions given by

$$
e_{n}:=\sqrt{\frac{2}{\pi}} \sin n x, n=1,2, \ldots
$$

In addition $\left(e_{n}\right)_{n \in \mathbb{N}}$ is a complete orthonormal basis in $X$ and

$$
T(t) x=\sum_{n=1}^{\infty} e^{-n^{2} t}<x, e_{n}>e_{n}
$$

for $x \in X$ and $t \geq 0$.

Now, we define an operator $A(t): D(A) \subset X \longrightarrow X$ by

$$
A(t) x(\zeta)=A x(\zeta)+b(t, \zeta) x(\zeta) .
$$

By assuming that $b(.,$.$) is continuous and that b(t, \zeta) \leq-\gamma(\gamma>0)$ for every $t \in \mathbb{R}$, $\zeta \in[0, \pi]$, it follows that the system

$$
\left\{\begin{array}{l}
u^{\prime}(t)=A(t) u(t), \quad t \geq s \\
u(s)=x \in X
\end{array}\right.
$$

has an associated evolution family given by

$$
\left.U(t, s) x(\zeta)=\left[T(t-s) \exp \left(\int_{s}^{t} b(\tau, \zeta)\right) d \tau\right) x\right](\zeta) .
$$

From this expression, it follows that $U(t, s)$ is a compact linear operator and that for every $s, t \in[0, T]$ with $t>s$

$$
\|U(t, s)\| \leq e^{-(\gamma+1)(t-s)}
$$

In addition, $A(t)$ satisfies the assumption $\mathcal{H}_{1}$ (see [3, 22]).

To rewrite the initial-boundary value problem (4.1) in the abstract form we assume the following:

i) $B: U \longrightarrow X$ is a bounded linear operator defined by

$$
B u(t)(\xi)=v(t, \xi), 0 \leq \xi \leq \pi, u \in L^{2}([0, T], U) .
$$

ii) The operator $W: L^{2}([0, T], U) \longrightarrow X$ defined by

$$
W u=\int_{0}^{T} S(T-s) v(t, \xi) d s
$$

has an inverse $W^{-1}$ and satisfies condition (H.5). For the construction of the operator $W$ and its inverse, see [26].

iii) The substitution operator $f:[0, T] \times X \longrightarrow X$ defined by $f(t, u)()=$. $f_{1}(t, u()$.$) is continuous and we impose suitable conditions on f_{1}$ to verify assumption $\mathcal{H}_{2}$.

iv) The substitution operator $g:[0, T] \times X \longrightarrow X$ defined by $g(t, u)()=$. $g_{1}(t, u()$.$) is continuous and we impose suitable conditions on g_{1}$ to verify assumptions $\mathcal{H}_{2}$ and $\mathcal{H}_{3}$. 
If we put

$$
\left\{\begin{array}{l}
x(t)(\zeta)=x(t, \zeta), t \in[0, T], \zeta \in[0, \pi] \\
x(t, \zeta)=\varphi(t, \zeta), t \in[-\tau, 0], \zeta \in[0, \pi]
\end{array}\right.
$$

then, the problem (4.1) can be written in the abstract form

$\left\{\begin{array}{l}d[x(t)+g(t, x(t-r(t)))]=\left[A(t) x(t)+f(t, x(t-\rho(t))] d t+\sigma(t) d B^{H}(t), 0 \leq t \leq T,\right. \\ x(t)=\varphi(t),-\tau \leq t \leq 0 .\end{array}\right.$

Furthermore, if we assume that the initial data $\varphi=\{\varphi(t):-\tau \leq t \leq 0\}$ satisfies $\varphi \in \mathcal{C}\left([-\tau, 0], \mathbb{L}^{2}(\Omega, X)\right)$, thus all the assumptions of Theorem 3.3 are fulfilled. Therefore, we conclude that the system (4.1) is controllable on $[-\tau, T]$.

\section{REFERENCES}

[1] P. Acquistapace, B. Terreni, A unified approach to abstract linear parabolic equations, Tend. Sem. Mat. Univ. Padova 78 (1987), 47-107.

[2] A. Anguraj, A. Vinodkumar, Existence, uniqueness and stability results of impulsive stochastic semilinear neutral functional differential equations with infinite delays, Electron. J. Qual. Theory Differ. Equ. Vol. 2009, No. 67 (2009), 1-13.

[3] D. Aoued, S. Baghli, Mild solutions for Perturbed evolution equations with infinite statedependent delay. Electronic Journal of Qualitative Theory of Differential Equations, No. 59 (2013), 1-24.

[4] G. Arthi, K. Balachandran, Controllability of second-order impulsive functional diferential equations with state-dependent delay, Bull. Korean Math. Soc., 48(6) (2011), 1271-1290.

[5] K. Balachandran, J. Y. Park, S. H. Park, Controllability of nonlocal impulsive quasilinear integrodifferential systems in Banach spaces, Rep. Math. Phys., 65 (2010), 247-257.

[6] K. Balachandran, R. Sathya, Controllability of nonlocal impulsive stochastic quasilinear integrodifferential systems, Electron. J. Qual. Theory Differ. Equ., 50 (2011), 1-16.

[7] P. Balasubramaniam, J. P. Dauer, Controllability of semilinear stochastic delay evolution equations in Hilbert spaces, International Journal of Mathematics and Mathematical Sciences, vol. 31, no. 3, (2002), 157-166.

[8] B. Boufoussi, S. Hajji, Neutral stochastic functional differential equation driven by a fractional Brownian motion in a Hilbert space. Statist. Probab. Lett. 82 (2012), 1549-1558.

[9] B. Boufoussi, S. Hajji, E. Lakhel, Functional differential equations in Hilbert spaces driven by a fractional Brownian motion. Afrika Matematika, Volume 23, Issue 2 (2011), 173-194.

[10] B. Boufoussi, S. Hajji, E. Lakhel. Time-dependent Neutral stochastic functional differential equation driven by a fractional Brownian motion in a Hilbert space. Preprint: arXiv:1401.2555 [math.PR].

[11] Y. K. Chang, A. Anguraj, M. Mallika Arjunan, Controllability of impulsive neutral functional differential inclusions with infinite delay in Banach spaces, Chaos Solitons and Fractals, 39 (2009), 1864-1876.

[12] T. Caraballo, M. J. Garrido-Atienza, T. Taniguchi, The existence and exponential behavior of solutions to stochastic delay evolution equations with a fractional Brownian motion. Nonlinear Analysis 74 ( 2011), 3671-3684.

[13] E. Lakhel, S. Hajji, Existence and Uniqueness of Mild Solutions to Neutral SFDEs driven by a Fractional Brownian Motion with Non-Lipschitz Coefficients. Journal of Num. Math. and Stochastics (To appear).

[14] J. Klamka, Stochastic controllability of linear systems with delay in control. Bull. Pol. Acad. Sci. Tech. Sci., 55 (2007), 23-29.

[15] A. N. Kolmogorov, Wienershe spiralen and einige andere interessante kurven im Hilbertschen raum, C. R. (Doklady) Acad. Sci. URSS (NS). 26 (1940), 115-118.

[16] B. B. Mandelbrot, J. W. Van Ness, Fractional Brownian motions, fractional noises and applications, SIAM Rev. 10 (4) (1968), 422-437.

[17] N. I. Mahmudov, Controllability of semilinear stochastic systems in Hilbert spaces, J. Math. Anal. Appl., 288, (1) (2003), 197-211.

[18] D. Nualart, The Malliavin Calculus and Related Topics, second edition. Springer-Verlag, Berlin. 2006. 
[19] Y. Ren, L. Hu, R. Sakthivel. Controllability of impulsive neutral stochastic functional differential inclusions with infinite delay, J. Comput. Appl. Math. 235 (8) (2011), 2603-2614.

[20] Y. Ren, D. Sun, Second-order neutral impulsive stochastic differential equations with delay. J. Math. Phys. 50 (2009), 702-709.

[21] Y. Ren, X. Cheng, R. Sakthivel, On time-dependent stochastic evolution equations driven by fractional Brownian motion in Hilbert space with finite delay. Mathematical methods in the Applied Sciences 2013.

[22] Y. Ren, Q. Zhou, L. Chen, Existence, uniqueness and stability of mild solutions for timedependent stochastic evolution equations with Poisson jumps and infinite delay J. Optim. Theory, 149 (2011) 315-331.

[23] R. Sakthivel, Complete controllability of stochastic evolution equations with jumps. Rep. Math. Phys., 68, 163-174.

[24] R. Sakthivel, Y. Ren, Complete controllability of stochastic evolution equations with jumps, Reports on Mathematical Physics, vol. 68, no. 2, (2011), 163-174.

[25] A. PAZY, Semigroups of Linear Operators and Applications to Partial Differential Equations. Applied Mathematical Sciences, vol. 44, Springer-Verlag, New York. 1983.

[26] M. D. Quinn , N. Carmichael, An approach to non linear control problems using fixed point methods, degree theory and pseudo-inverses, Numer. Funct. Anal. Optim. 7 (1984-1985) 1977-219.

[27] D. Xu , Z.Yang, Exponential stability of nonlinear impulsive neutral differential equations with delays, Nonlinear Anal. 67 (5) (2006), 1426-1439. 